\title{
Superscaling and neutral current quasielastic neutrino-nucleus scattering beyond the relativistic Fermi gas model
}

\author{
A. N. Antonov, ${ }^{1}$ M. V. Ivanov, ${ }^{1}$ M. B. Barbaro, ${ }^{2}$ J. A. Caballero, ${ }^{3}$ E. Moya de Guerra,,${ }^{4,5}$ and M. K. Gaidarov ${ }^{1,4}$ \\ ${ }^{1}$ Institute for Nuclear Research and Nuclear Energy, Bulgarian Academy of Sciences, Sofia 1784, Bulgaria \\ ${ }^{2}$ Dipartimento di Fisica Teorica, Università di Torino and INFN, Sezione di Torino, Via P. Giuria 1, I-10125 Torino, Italy \\ ${ }^{3}$ Departamento de Física Atómica, Molecular y Nuclear, Universidad de Sevilla, Apdo. 1065, E-41080 Sevilla, Spain \\ ${ }^{4}$ Instituto de Estructura de la Materia, CSIC, Serrano 123, E-28006 Madrid, Spain \\ ${ }^{5}$ Departamento de Fisica Atomica, Molecular y Nuclear, Facultad de Ciencias Fisicas, Universidad Complutense de Madrid, \\ E-28040 Madrid, Spain \\ (Received 4 April 2007; published 27 June 2007)
}

\begin{abstract}
The superscaling analysis is extended to include quasielastic (QE) scattering via the weak neutral current (NC) of neutrinos and antineutrinos from nuclei. The scaling function obtained within the coherent density fluctuation model (CDFM) [used previously in calculations of QE inclusive electron and charge-changing (CC) neutrino scattering] is applied to neutral current neutrino and antineutrino scattering with energies of $1 \mathrm{GeV}$ from ${ }^{12} \mathrm{C}$ with a proton and neutron knockout ( $u$-channel inclusive processes). The results are compared with those obtained using the scaling function from the relativistic Fermi gas model and the scaling function as determined from the superscaling analysis (SuSA) of QE electron scattering.
\end{abstract}

DOI: 10.1103/PhysRevC.75.064617

PACS number(s): 25.30.Pt, 23.40.Bw, 24.10.-i, 21.60.-n

\section{INTRODUCTION}

The studies of the vast amount of inclusive electron scattering world data have shown the existence of $y$-scaling [1-10] and superscaling (based on $\psi^{\prime}$-scaling variable) (see, e.g., [10-21]) phenomena. A very weak dependence of the reduced cross section on the momentum transfer $q$ (scaling of the first kind) has been observed at excitation energies below the quasielastic peak for large enough $q$. Scaling of the second kind (i.e., no dependence of the reduced cross section on the mass number) has been found to be excellent in the same region. When both types of scaling occur one says that the reduced cross sections exhibit superscaling. It has been shown (e.g., in [16-20]) that the superscaling phenomenon is related to the specific high-momentum tail of the nucleon momentum distribution $n(k)$ at momenta $k>2 \mathrm{fm}^{-1}$ which is similar for all nuclei and is due to the short-range and tensor correlations in the nuclear medium.

It has been observed also that above the quasielastic (QE) peak the scaling of the second kind is good, but scaling of the first kind is violated. The latter occurs due to the excitation of a nucleon in the nucleus to a delta-resonance which subsequently decays into a nucleon and a pion (e.g., [15,22]). Additionally, meson exchange currents are known to violate the scaling behavior [23-26], although their effects appear not to be the dominant ones [27].

In $[10,11]$ the theoretical concept of superscaling has been introduced within the relativistic Fermi gas (RFG) model. As pointed out in [13], however, the actual dynamical physical reason of the superscaling is more complex than that provided by the RFG model. For instance, the QE scaling function in the RFG model is $f_{\mathrm{RFG}}^{\mathrm{QE}}\left(\psi^{\prime}\right)=0$ for $\psi^{\prime} \leqslant-1$, whereas the experimental scaling function $f^{\mathrm{QE}}\left(\psi^{\prime}\right)$ extends to large negative values of $\psi^{\prime}$ up to $\psi^{\prime} \approx-2$ in the data for $\left(e, e^{\prime}\right)$ processes. Thus, the necessity to consider the superscaling in theoretical methods which go beyond the RFG model has arisen. One of them is the coherent density fluctuation model (e.g., [28,29]) being a natural extension of the Fermi gas case to realistic finite nuclear systems. As pointed out in [16-19], in the coherent density fluctuation model both basic quantities, density and momentum distributions are responsible for the scaling and superscaling behavior in nuclei. The QE scaling function in the CDFM $f\left(\psi^{\prime}\right)$ agrees with the available experimental data for $\psi^{\prime}<0$, including $\psi^{\prime} \lesssim-1$.

In [30] the superscaling analyses of the electron scattering for energies of several hundred $\mathrm{MeV}$ to a few $\mathrm{GeV}$ have been extended to include not only QE processes but also those in which $\Delta$-excitation dominates. Both QE- and $\Delta$-region scaling functions $f^{\mathrm{QE}}\left(\psi^{\prime}\right)$ and $f^{\Delta}\left(\psi_{\Delta}^{\prime}\right)$ have been deduced in [30] from phenomenological fits to the data for electronnuclei scattering cross sections. Generally, the theoretical microscopical construction of the scaling function should take into account final-state interactions (FSI). By using a relativistic mean field for the final states, in [31,32] a scaling function with asymmetric shape has been obtained being in agreement with the experimental scaling function. Also an asymmetrical scaling function in accordance with data has been obtained recently [33] within a semi-relativistic approach, based on improved nonrelativistic expansions, but with FSI described with the Dirac equation-based potential. The asymmetry of the QE scaling function in the CDFM has been introduced in a phenomenological way [19] accounting for the role of FSI.

The analyses of the superscaling phenomenon and the present knowledge of inclusive electron scattering off nuclei have induced studies of neutrino scattering from nuclei on the same basis. This makes it possible to explore fundamental questions of neutrino reactions and neutrino oscillations in relation to hypothesis of nonzero neutrino masses [34]. In [30] (see also [31,35]) the scaling ideas have been inverted: given the scaling functions one can just multiply by the elementary charge-changing neutrino cross sections to obtain 
corresponding $\mathrm{CC}$ neutrino and antineutrino cross sections on nuclei for intermediate to high energies in the same region of excitation. In [27] the scaling and superscaling ideas have been carried a step further to include neutral current neutrino and antineutrino scattering cross sections for scattering from ${ }^{12} \mathrm{C}$, namely for reactions ${ }^{12} \mathrm{C}(\nu, p) v X,{ }^{12} \mathrm{C}(\bar{v}, p) \bar{v} X$ involving proton knockout and ${ }^{12} \mathrm{C}(v, n) v X,{ }^{12} \mathrm{C}(\bar{v}, n) \bar{v} X$ involving neutron knockout in the $\mathrm{QE}$ regime. $\mathrm{A}$ number of other theoretical considerations (e.g., [36-46]) have been devoted to studies of both neutral- (e.g., [36-39]) and charge-changing (e.g., [37-46]) neutrino-nucleus scattering.

In [19] the QE- and $\Delta$-region scaling functions obtained in the CDFM and within the modified parameter-free theoretical approach [47] based on the light-front dynamics method (LFD) (e.g., $[48,49])$ have been applied to describe the experimental data on differential cross sections of QE inclusive electron scattering as well as to analyze charge-changing neutrino scattering on the ${ }^{12} \mathrm{C}$ nucleus for energies of the incident particles from 1 to $2 \mathrm{GeV}$. It has been shown that the results for electron scattering on ${ }^{12} \mathrm{C}$ are close to those of the superscaling analysis $[15,30]$ and are quite different from the RFG results, whereas the almost symmetric CDFM scaling function leads to cross sections that are similar to the results of the RFG model.

The aim of the present work is to extend the application of the CDFM scaling function to calculations of neutral current neutrino and antineutrino scattering cross sections from nuclei, e.g., to consider reactions on ${ }^{12} \mathrm{C}$ as those in [27] and mentioned above with proton and neutron knockout in the $\mathrm{QE}$ region. We should note that, as it has been considered in $[27,50]$, when one has an incident lepton, a scattering with exchange of a $\gamma, W^{ \pm}$or $Z^{0}$ and the scattered lepton (i.e., a charged lepton) is detected, the $t$-channel exchange of the corresponding boson is controlled. When, however, the scattered lepton is a (not detected) neutrino or antineutrino, and a knocked-out nucleon is detected, then the kinematics of the $u$-channel are controlled. We also follow [27] on the formalism for cross sections and, what is more crucial, in what concerns the scaling ideas that interrelate the $t$ - and $u$-channel processes.

The paper is organized in the following way. The theoretical scheme is given in Sec. II. It includes the formalism for $u$-channel scattering including briefly the kinematics, cross sections and scaling as well as the main relationships of the CDFM used in the superscaling analysis. The results of $\mathrm{NC}$ neutrino and antineutrino scattering cross sections on ${ }^{12} \mathrm{C}$ are presented and discussed in Sec. III. The conclusions are summarized in Sec. IV.

\section{THE THEORETICAL SCHEME}

\section{A. Kinematics: Cross sections and scaling}

We consider the semileptonic quasifree scattering from nuclei in Born approximation, assuming that the inclusive cross sections are well represented by the sum of the integrated semi-inclusive proton and neutron emission cross sections [27]. The kinematics for semileptonic nucleon knockout reac-

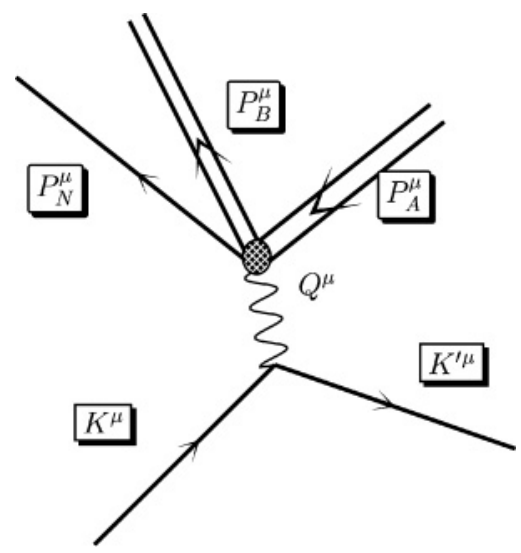

FIG. 1. The kinematics for semi-leptonic nucleon knockout reactions in the one-boson-exchange approximation.

tions in the one-boson-exchange approximation is presented in Fig. 1.

A lepton with four-momentum $K^{\mu}=(\epsilon, \mathbf{k})$ scatters to another lepton with four-momentum $K^{\prime \mu}=\left(\epsilon^{\prime}, \mathbf{k}^{\prime}\right)$, exchanging a vector boson with four-momentum $Q^{\mu}=K^{\mu}-K^{\prime \mu}$. The lepton energies are $\epsilon=\sqrt{m^{2}+k^{2}}$ and $\epsilon^{\prime}=\sqrt{m^{\prime 2}+k^{\prime 2}}$, where the masses of the initial and final lepton $m$ and $\left(m^{\prime}\right)$ are assumed to be equal to zero for $\mathrm{NC}$ neutrino scattering. In the laboratory system the initial nucleus being in its ground state has a four-momentum $P_{A}^{\mu}=\left(M_{A}^{0}, 0\right)$, while the final hadronic state corresponds to a proton or neutron with four-momentum $P_{N=p \text { or } n}^{\mu}=\left(E_{N}, \mathbf{p}_{N}\right)$ and an unobserved residual nucleus with four-momentum $P_{B}^{\mu}=\left(E_{B}, \mathbf{p}_{B}\right)$. Usually the missing momentum $\mathbf{p} \equiv-\mathbf{p}_{B}$ and the excitation energy $\mathcal{E} \equiv E_{B}-E_{B}^{0}$, with $E_{B}^{0}=\sqrt{\left(M_{B}^{0}\right)^{2}+p^{2}}$ are introduced, $M_{B}^{0}$ being the ground-state mass of the daughter nucleus. We assume for $\mathrm{NC}$ neutrino scattering that the neutrino beam momentum is specified and the outgoing proton (or neutron) is detected (for details see $[12,13,27])$. The exchanged four-momentum in the $u$-channel is defined as

$$
Q^{\prime \mu} \equiv K^{\mu}-P_{N}^{\mu}=\left(\omega^{\prime}, \mathbf{q}^{\prime}\right) .
$$

Details on the kinematics and integration limits involved in $\mathrm{NC}$ neutrino-nucleus scattering are given in [27].

The usual procedure for calculating the $\left(l, l^{\prime} N\right)$ cross section includes the plane wave impulse approximation (PWIA) and integrations over all unconstrained kinematic variables. It is shown in [27] that the inclusive cross section in the $u$-channel can be written after some approximations in the following form:

$$
\frac{d \sigma}{d \Omega_{N} d p_{N}} \approx \bar{\sigma}_{s n}^{(u)} F\left(y^{\prime}, q^{\prime}\right),
$$

where

$$
F\left(y^{\prime}, q^{\prime}\right) \equiv \int_{\mathcal{D}_{u}} p d p \int \frac{d \mathcal{E}}{E} \Sigma \simeq F\left(y^{\prime}\right),
$$


provided the effective NC single nucleon (s.n.) cross section

$$
\begin{aligned}
\bar{\sigma}_{\mathrm{s} . \mathrm{n} .}^{(u)}= & \frac{1}{32 \pi \epsilon} \frac{1}{q^{\prime}}\left(\frac{p_{N}^{2}}{E_{N}}\right) g^{4} \int_{0}^{2 \pi} \frac{d \phi^{\prime}}{2 \pi} l_{\mu \nu}\left(\mathbf{k}, \mathbf{k}^{\prime}\right) \\
& \times w^{\mu \nu}\left(\mathbf{p}, \mathbf{p}_{N}\right) D_{V}\left(Q^{2}\right)^{2}
\end{aligned}
$$

is almost independent of $(p, \mathcal{E})$ for constant $\left(k, p_{N}, \theta_{k p_{N}}\right)$. In Eq. (4) $l_{\mu \nu}$ and $w^{\mu \nu}$ are the leptonic and s.n. hadronic tensor, respectively, and $D_{V}\left(Q^{2}\right)$ is the vector boson propagator [27]. In Eq. (2) $y^{\prime}$ is the scaling variable naturally arising in the $u$-scattering kinematics, analogous to the usual $y$-scaling variable for $t$-scattering. The scaling function $F\left(y^{\prime}\right)$ obtained within a given approach can be used to predict realistic NC cross sections. Assuming that the domains of integration $\mathcal{D}_{u}$ (in the $u$-channel) and $\mathcal{D}_{t}$ (in the $t$-channel) are the same or very similar, the results for the scaling function obtained in the case of inclusive electron scattering (where $\mathcal{D}_{t}$ works) can be used in the case of NC neutrino reactions. It is pointed out in [27] that $\mathcal{D}_{t}$ and $\mathcal{D}_{u}$ differ significantly only at large $\mathcal{E}$ (also at large $p$, but there one believes that the semi-inclusive cross sections are negligible). So, given that the semi-inclusive cross sections are dominated by their behavior at low $\mathcal{E}$ and low $p$, one expects the results of the integrations in the $t$ - and $u$-channel to be very similar, and thus the scaling functions will be essentially the same in both cases.

The RFG $u$-channel $\psi$-variable is introduced in the form [27]

$$
\psi_{\mathrm{RFG}}^{(u)}=s \sqrt{\frac{m_{N}}{T_{F}}}\left[\sqrt{1+\left(\frac{y_{\mathrm{RFG}}^{(u)}}{m_{N}}\right)^{2}}-1\right]^{1 / 2},
$$

where

$$
y_{\mathrm{RFG}}^{(u)}=s \frac{m_{N}}{\tau^{\prime}}\left[\lambda^{\prime} \sqrt{\tau^{\prime 2} \rho^{\prime 2}+\tau^{\prime}}-\kappa^{\prime} \tau^{\prime} \rho^{\prime}\right]
$$

is the RFG $y$-scaling variable for the $u$-channel and corresponds to the minimum momentum required for a nucleon to participate in the $\mathrm{NC}$ neutrino-nucleus scattering. The dimensionless kinematic quantities in Eq. (6) are given by $\kappa^{\prime} \equiv q^{\prime} / 2 m_{N}, \lambda^{\prime} \equiv \omega^{\prime} / 2 m_{N}, \tau^{\prime}=\kappa^{\prime 2}-\lambda^{\prime 2}$, and defined $\rho^{\prime} \equiv 1-\frac{1}{4 \tau^{\prime}}\left(1-m^{\prime 2} / m_{N}^{2}\right)$. The sign $s$ is

$$
s \equiv \operatorname{sgn}\left\{\frac{1}{\tau^{\prime}}\left[\lambda^{\prime} \sqrt{\tau^{\prime 2} \rho^{\prime 2}+\tau^{\prime}}-\kappa^{\prime} \tau^{\prime} \rho^{\prime}\right]\right\} .
$$

The physical meaning of $\psi_{\mathrm{RFG}}^{(u)}$ is the minimum kinetic energy of the nucleon participating in the reaction. The RFG scaling function is found to be

$$
F_{\mathrm{RFG}}\left(\psi_{\mathrm{RFG}}^{(u)}\right)=\frac{3}{4} k_{F}\left(1-\psi_{\mathrm{RFG}}^{(u) 2}\right) \Theta\left(1-\psi_{\mathrm{RFG}}^{(u) 2}\right) .
$$

As noted in [27], if the s.n. cross section is smoothly varying within the $(p, \mathcal{E})$ integration region, the differential cross section in the RFG can be factorized as shown in Eq. (2) with the scaling function from Eq. (8). In this work, however, we use the scaling function calculated in the CDFM model which is beyond the RFG model (see Sec. II B).
The basic relationships used to calculate the s.n. cross sections are given in [27]. This concerns the leptonic and hadronic tensors and the response and structure functions. The Höhler parametrization for the single-nucleon form factors [51] is used, ignoring the strangeness content of the nucleon.

\section{B. QE scaling function in the CDFM}

In this subsection we present briefly the main expressions concerning the $\mathrm{QE}$ scaling function $f^{\mathrm{QE}}\left(\psi^{\prime}\right)$ within the CDFM $[28,29]$ (which is a natural extension of the RFG model). This function was obtained (see [19] and references therein) in two ways that were shown to be equivalent: on the basis of the local density distribution $[\rho(r)]$ and on the basis of the nucleon momentum distribution $[n(k)]$. Generally, the total CDFM scaling function is expressed by the sum of the proton $f_{p}^{\mathrm{QE}}\left(\psi^{\prime}\right)$ and neutron $f_{n}^{\mathrm{QE}}\left(\psi^{\prime}\right)$ scaling functions, which are determined by the proton and neutron densities $\rho_{p}(r)$ and $\rho_{n}(r)$ (or by corresponding momentum distributions), respectively:

$$
f^{\mathrm{QE}}\left(\psi^{\prime}\right)=\frac{1}{A}\left[Z f_{p}^{\mathrm{QE}}\left(\psi^{\prime}\right)+N f_{n}^{\mathrm{QE}}\left(\psi^{\prime}\right)\right] .
$$

The CDFM scaling function gives a good description of the superscaling phenomenon. In the consideration in [16-18] it has a symmetric form for negative and positive values of $\psi^{\prime}$. The maximum value of $f^{\mathrm{QE}}\left(\psi^{\prime}\right)$ in CDFM (and in RFG) is 3/4 whereas, however, the empirical "universal" scaling function extracted in [30] reaches 0.6 and has a markedly asymmetric shape. Also, an asymmetric shape of $f\left(\psi^{\prime}\right)$ has been found in $[31,32]$ from calculations for $\left(e, e^{\prime}\right)$ and $(\nu, \mu)$ reactions based on the relativistic impulse approximation with FSI using the relativistic mean-field potential.

In [19] we limited our CDFM approach to phenomenology when considering the asymmetric shape and the maximum value of the QE $f\left(\psi^{\prime}\right)$. The role of all the effects that lead to asymmetry has been simulated by imposing asymmetry on the RFG scaling function (and, correspondingly, on the CDFM one) by introducing a parameter which gives the correct maximum value of the scaling function $\left(c_{1}\right.$ in our notations below) and also an asymmetric tail in $f^{\mathrm{QE}}\left(\psi^{\prime}\right)$ for $\psi^{\prime} \geqslant 0$. The proton and neutron scaling functions in Eq. (9) are presented as sums of scaling functions for negative $\left[f_{p(n), 1}^{\mathrm{QE}}\left(\psi^{\prime}\right)\right]$ and positive $\left[f_{p(n), 2}^{\mathrm{QE}}\left(\psi^{\prime}\right)\right]$ values of $\psi^{\prime}$ :

$$
f_{p(n)}^{\mathrm{QE}}\left(\psi^{\prime}\right)=f_{p(n), 1}^{\mathrm{QE}}\left(\psi^{\prime}\right)+f_{p(n), 2}^{\mathrm{QE}}\left(\psi^{\prime}\right) .
$$

In Eq. (10)

$$
\begin{aligned}
f_{p(n), 1}^{\mathrm{QE}}\left(\psi^{\prime}\right) & =\int_{0}^{\alpha_{p(n)} /\left(k_{F}^{p(n)}\left|\psi^{\prime}\right|\right)} d R\left|F_{p(n)}(R)\right|^{2} f_{\mathrm{RFG}, 1}^{p(n)}\left(\psi^{\prime}(R)\right), \\
\psi^{\prime} & \leqslant 0 \\
f_{p(n), 2}^{\mathrm{QE}}\left(\psi^{\prime}\right) & =\int_{0}^{c_{2} \alpha_{p(n)} /\left(k_{F}^{p(n)} \psi^{\prime}\right)} d R\left|F_{p(n)}(R)\right|^{2} f_{\mathrm{RFG}, 2}^{p(n)}\left(\psi^{\prime}(R)\right), \\
\psi^{\prime} & \geqslant 0,
\end{aligned}
$$


where

$$
f_{\mathrm{RFG}, 1}^{p(n)}\left(\psi^{\prime}(R)\right)=c_{1}\left[1-\left(\frac{k_{F}^{p(n)} R\left|\psi^{\prime}\right|}{\alpha_{p(n)}}\right)^{2}\right], \quad \psi^{\prime} \leqslant 0
$$

and

$$
f_{\mathrm{RFG}, 2}^{p(n)}\left(\psi^{\prime}(R)\right)=c_{1} \exp \left[-\frac{k_{F}^{p(n)} R \psi^{\prime}}{c_{2} \alpha_{p(n)}}\right], \quad \psi^{\prime} \geqslant 0 .
$$

In Eqs. (11) and (12) the proton and neutron weight functions are obtained from the corresponding proton and neutron densities

$$
\begin{aligned}
\left|F_{p(n)}(R)\right|^{2} & =-\left.\frac{4 \pi R^{3}}{3 Z(N)} \frac{d \rho_{p(n)}(r)}{d r}\right|_{r=R}, \\
\alpha_{p(n)} & =\left[\frac{9 \pi Z(N)}{4}\right]^{1 / 3}, \\
\int_{0}^{\infty} \rho_{p(n)}(\mathbf{r}) d \mathbf{r} & =Z(N)
\end{aligned}
$$

and the Fermi-momentum for the protons and neutrons can be calculated using the expression

$$
k_{F}^{p(n)}=\alpha_{p(n)} \int_{0}^{\infty} d R \frac{1}{R}\left|F_{p(n)}(R)\right|^{2} .
$$

The functions are normalized as follows:

$$
\begin{aligned}
& \int_{0}^{\infty}\left|F_{p(n)}(R)\right|^{2} d R=1, \\
& \int_{-\infty}^{\infty} f_{p(n)}^{\mathrm{QE}}\left(\psi^{\prime}\right) d \psi^{\prime}=1 .
\end{aligned}
$$

From the normalization of the total QE scaling function

$$
\int_{-\infty}^{\infty} f^{\mathrm{QE}}\left(\psi^{\prime}\right) d \psi^{\prime}=1
$$

one can get relationship between $c_{2}$ and $c_{1}$ :

$$
c_{2}=\frac{1-\frac{2}{3} c_{1}}{c_{1}\left(\frac{e-1}{e}\right)} \simeq \frac{1-\frac{2}{3} c_{1}}{0.632 c_{1}} \text {. }
$$

The value of $c_{2}=1$ corresponds to $c_{1}=\frac{3}{4}$. The asymmetry of the scaling function increases with the decrease of $c_{1}$ from $\frac{3}{4}$.

In [19] also a parabolic form of $f_{\mathrm{RFG}, 2}^{p(n)}\left(\psi^{\prime}(R)\right)$

$$
f_{\mathrm{RFG}, 2}^{p(n)}\left(\psi^{\prime}(R)\right)=c_{1}\left[1-\left(\frac{k_{F}^{p(n)} R \psi^{\prime}}{c_{2} \alpha_{p(n)}}\right)^{2}\right], \psi^{\prime} \geqslant 0
$$

instead the exponential one in Eq. (14) was considered. In this case $c_{2}=\frac{3}{2 c_{1}}-1$.

As already mentioned, the QE- and $\Delta$-scaling functions obtained in the CDFM and in the LFD approach were applied in [19] to describe experimental data on differential cross sections of inclusive electron scattering by ${ }^{12} \mathrm{C}$ at large
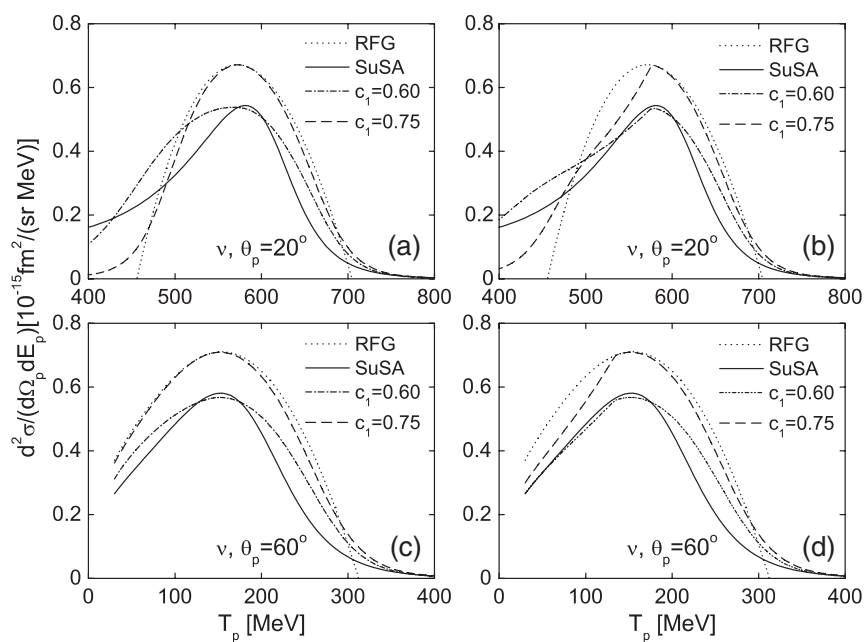

FIG. 2. Quasielastic differential cross section for neutral current neutrino scattering at $1 \mathrm{GeV}$ from ${ }^{12} \mathrm{C}$ for proton knockout at $\theta_{p}=20^{\circ}$ (a,b) and $60^{\circ}(\mathrm{c}, \mathrm{d})$ using the CDFM scaling function [Eqs. (9)-(13), (23) for (a,c) and Eqs. (9)-(14) for (b,d)] with $c_{1}=0.60$ (dash-dotted line) and $c_{1}=0.75$ (dashed line). The RFG results are given by dotted line and the results using the empirical scaling function [27] are presented by solid line (SuSA).

energies and transferred momenta as well as to calculate QE charge-changing neutrino-nuclei reaction cross sections. It was shown in the case of the electron scattering that the results obtained when asymmetric scaling function $f^{\mathrm{QE}}\left(\psi^{\prime}\right)$ $\left(c_{1}^{\mathrm{QE}}=0.63\right)$ with $f_{\mathrm{RFG}, 2}^{p(n)}\left(\psi^{\prime}(R)\right)$ from Eq. (23) is used agree with the data in cases when the transferred momentum in the position of the maximum of the QE peak extracted from data $\left(\omega_{\exp }^{\mathrm{QE}}\right)$ is $q_{\exp }^{\mathrm{QE}}<450 \mathrm{MeV} / c \approx 2 k_{F}$ and underestimate them when $q_{\exp }^{\mathrm{QE}} \geqslant 450 \mathrm{MeV} / c$ in the region close to the QE peak. The almost symmetric scaling function $f^{\mathrm{QE}}\left(\psi^{\prime}\right)\left(c_{1}^{\mathrm{QE}}=0.72\right)$ leads to results in agreement with the data in the region of the QE peak in cases when $q_{\exp }^{\mathrm{QE}} \geqslant 450 \mathrm{MeV} / c$, whereas the data are overestimated in cases where $q_{\exp }^{\mathrm{QE}}<450 \mathrm{MeV} / c$. As can be seen in Sec. III, the use of the exponential form of $f_{\mathrm{RFG}, 2}^{p(n)}\left(\psi^{\prime}(R)\right)$ [Eq. (14)] instead of the parabolic one [Eq. (23)] was imposed by the aim for a better description of the experimental data for the quasielastic scaling function $f^{\mathrm{QE}}\left(\psi^{\prime}\right)$ for $\psi^{\prime} \geqslant 0$ [see Fig. 6 for $f^{\mathrm{QE}}\left(\psi^{\prime}\right)$ in the case of ${ }^{12} \mathrm{C}$ nucleus]. In the cases of $\mathrm{CC}$ neutrino and antineutrino $\left(v_{\mu}, \mu^{-}\right)$and $\left(\bar{v}_{\mu}, \mu^{+}\right)$reactions on ${ }^{12} \mathrm{C}$ for energies of the incident particles from 1 to $2 \mathrm{GeV}$ the results obtained by using the asymmetric CDFM scaling function $f^{\mathrm{QE}}\left(\psi^{\prime}\right)\left(c_{1}^{\mathrm{QE}}=0.63\right)$ are close to those of SuSA $[15,30]$ and are different from the RFG model results, whereas the almost symmetric CDFM scaling function $f^{\mathrm{QE}}\left(\psi^{\prime}\right)\left(c_{1}^{\mathrm{QE}}=0.72\right)$ leads to cross sections that are similar to the results of the RFG model.

\section{RESULTS OF CALCULATIONS AND DISCUSSION}

In this section we present in Figs. 2-5 the results of the calculations of the cross sections of neutral current neutrino 

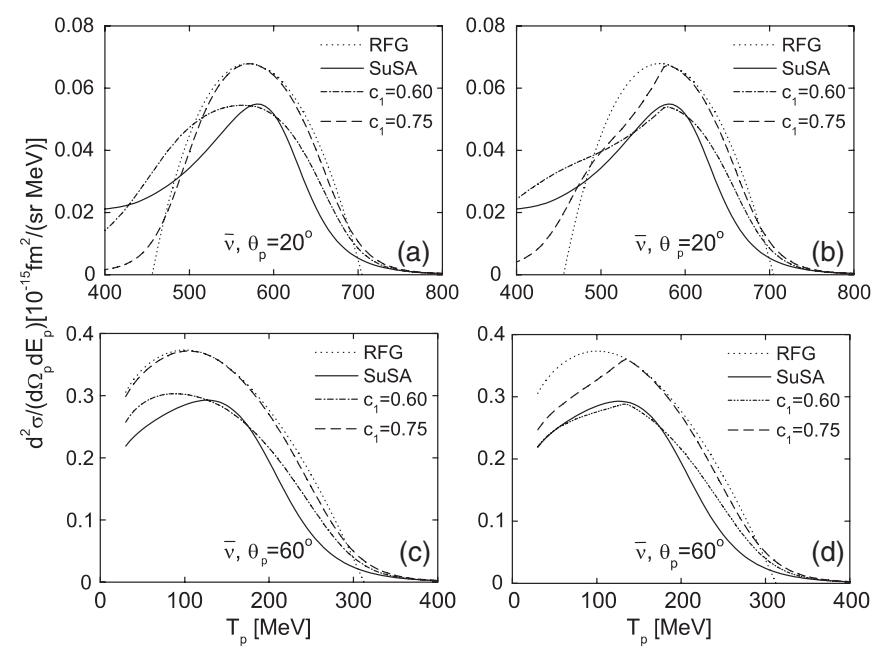

FIG. 3. The same as in Fig. 2 for neutral current antineutrino scattering.

(Figs. 2 and 4) and antineutrino (Figs. 3 and 5) scattering at $1 \mathrm{GeV}$ from ${ }^{12} \mathrm{C}$ with a knockout of proton (Figs. 2 and 3 ) and neutron (Figs. 4 and 5) as a function of the kinetic energy of the ejected nucleon. The calculations are performed for two values of the proton or neutron angle, namely $20^{\circ}(a, b)$ and $60^{\circ}(\mathrm{c}, \mathrm{d})$.

The two ingredients of the cross section, namely the s.n. cross section and the QE scaling function, are calculated according to the theoretical scheme presented in Secs. II A and II B, correspondingly. The NC neutrino and antineutrino scattering cross sections are calculated using Eqs. (2)-(4), while for the s.n. cross sections we followed the consideration in [27]. The CDFM QE scaling function for the ${ }^{12} \mathrm{C}$ nucleus was calculated by means of Eqs. (9)-(23) using in Eq. (15) the charge density of ${ }^{12} \mathrm{C}$ and assuming that the proton and neutron densities are the same. We used a symmetrized Fermi-type density distribution [52] with the following values of the half-radius $R_{1 / 2}$ and diffuseness $b$ parameters: $R_{1 / 2}=$ $2.470 \mathrm{fm}$ and $b=0.420 \mathrm{fm}$. These parameter values lead to
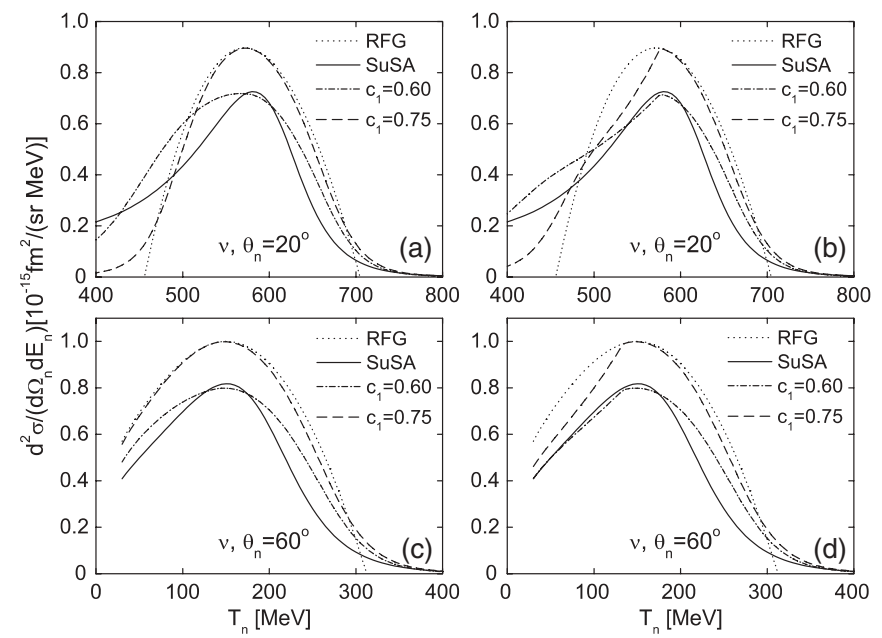

FIG. 4. The same as in Fig. 2 for neutral current neutrino scattering showing the neutron knockout case.
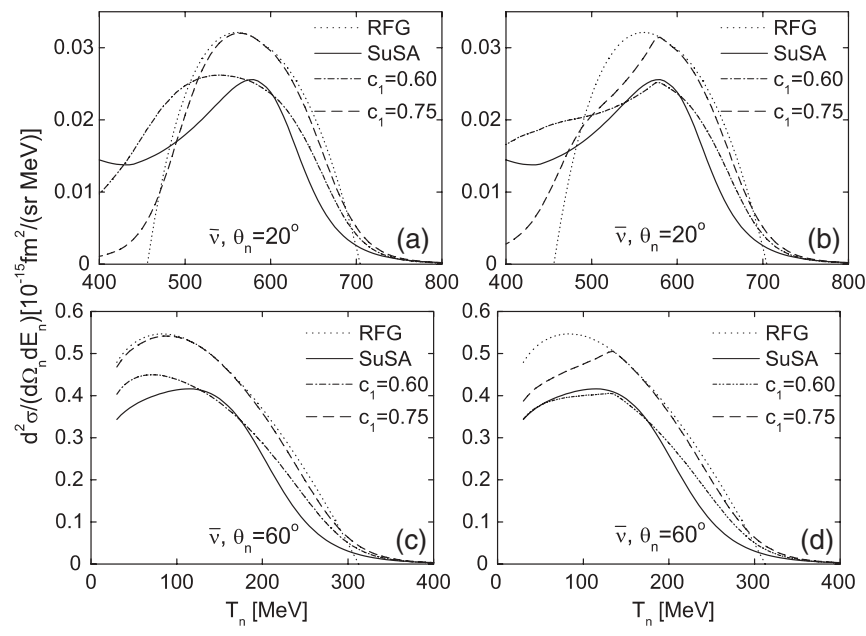

FIG. 5. The same as in Fig. 2 for neutral current antineutrino scattering showing the neutron knockout case.

charge rms radius equal to $2.47 \mathrm{fm}$ which coincides with the experimental one [53].

In Figs. 6(a) and 6(b) we present the quasielastic CDFM scaling function $f^{\mathrm{QE}}\left(\psi^{\prime}\right)$ for ${ }^{12} \mathrm{C}$ in comparison with the experimental data taken from [30], with the RFG result and with the SuSA result. The results for the QE scaling function using the parabolic form of the RFG scaling function for $\psi^{\prime} \geqslant 0$, [Eqs. (9)-(13), (23)] are given in Fig. 6(a), while those obtained using the exponential form of the RFG scaling function for $\psi^{\prime} \geqslant 0$ [Eqs. (9)-(14)] are given in Fig. 6(b). The CDFM scaling function is given in Figs. 6(a) and 6(b) for two values of the parameter $c_{1}: c_{1}=0.75$ and 0.60 . In the case of $c_{1}=0.75 f^{\mathrm{QE}}\left(\psi^{\prime}\right)$ is symmetric, while in the case with $c_{1}=0.60$ it is asymmetric. As can be seen in both cases the scaling functions calculated using $c_{1}=0.60$ are in better agreement with the empirical data. This is true even in the interval $\psi^{\prime}<-1$, whereas in the RFG model $f_{\mathrm{RFG}}\left(\psi^{\prime}\right)=0$ for $\psi^{\prime} \leqslant-1$.

The calculations of the quasielastic differential cross sections for neutral current neutrino and antineutrino scattering
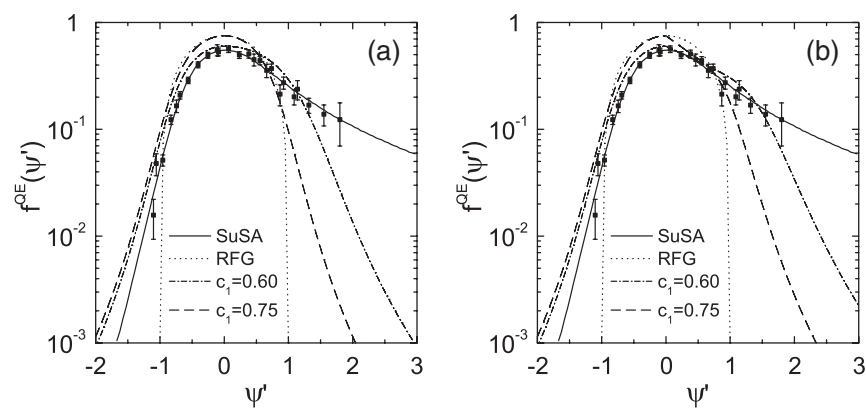

FIG. 6. (a) The quasielastic scaling function $f^{\mathrm{QE}}\left(\psi^{\prime}\right)$ for ${ }^{12} \mathrm{C}$ calculated in the CDFM using Eqs. (9)-(13), (23) with $c_{1}=0.60$ (dash-dotted line) and $c_{1}=0.75$ (dashed line) in comparison with the result of the RFG model (dotted line) and with the results from the SuSA (solid line). The experimental data (black squares) are taken from [30]; (b) the same as in (a) with the CDFM scaling function calculated by using Eqs. (9)-(14). 
in the proton and neutron knockout cases whose results are presented in Figs. 2-5, are performed by means of the CDFM QE scaling function in both cases [using Eq. (14) or Eq. (23) for $f_{\mathrm{RFG}, 2}^{p(n)}\left(\psi^{\prime}(R)\right]$ in Eqs. (9)-(13).

We would like to note the following features of the results. Firstly, it can be seen that our results by using $c_{1}=0.60$ [i.e., asymmetric $f^{\mathrm{QE}}\left(\psi^{\prime}\right)$ ] are close to those obtained in [27] from the SuSA showing a tail for larger values of the kinetic energy $T_{p(n)}$ in contrast to the RFG result. When using $c_{1}=0.75$ [i.e., symmetric $\left.f^{\mathrm{QE}}\left(\psi^{\prime}\right)\right]$ our cross sections are close to those from the RFG model. Their maxima are with larger magnitude than those in the case with $c_{1}=0.60$ and in SuSA and their slopes are steeper at large $T_{p(n)}$.

Secondly, the use of the exponential form of $f_{\mathrm{RFG}, 2}^{p(n)}\left(\psi^{\prime}(R)\right)$ [cases (b) and (d) in Figs. 2-5] leads to a sharper slope of the cross sections in comparison with the case of parabolic form [cases (a) and (c)] for $T_{p(n)}$ smaller than those in the maximum.

Thirdly, it can be seen from Figs. 2-5 that, similarly to the results in [27], the shapes of the cross sections for proton and neutron knockout are very similar. However, the magnitudes are somewhat different. For instance, the magnitude of the maximum of the NC cross section of neutrino scattering is much larger than that for antineutrino scattering cross section. This difference is around an order of magnitude in the case of proton knockout at $\theta_{p}=20^{\circ}$, and it is even larger in the case of neutron knockout at $\theta_{n}=20^{\circ}$. For $\theta_{p}$ and $\theta_{n}$ equal to $60^{\circ}$ these differences are smaller, around a factor of 2 .

Fourthly, except for antineutrinos at forward angles, the neutron knockout results are 30-50\% higher than the proton knockout. As noted in [27], this occurs because (in the absence of strangeness) both the vector and the axial-vector contributions are larger for neutrons than for protons, and they sum up.

\section{CONCLUSIONS}

In our previous work [19] we applied the superscaling analysis and scaling functions obtained within the CDFM and LFD approach to inclusive electron scattering as well as to charge-changing neutrino and antineutrino reactions at energies between 1 and $2 \mathrm{GeV}$ from the ${ }^{12} \mathrm{C}$ nucleus. The scaling functions describe well the superscaling phenomenon below the QE peak. In [19] the scaling function for the $\Delta$ region was constructed and a good representation of inclusive electron scattering cross sections data up to at least the peak of the $\Delta$-region was obtained. The required asymmetry (with a long tail extending to high energy loss) of the CDFM scaling function was introduced in a phenomenological way.

In the present work we extend the application of CDFM scaling functions to calculate differential cross sections of neutral current neutrino-(antineutrino-)nucleus scattering at intermediate-to-high energies. We construct asymmetric scaling functions within CDFM taking into account the deviation from experiment of the RFG scaling function at $\psi^{\prime}=0$ [see Eqs. (9) to (12)]. Two different asymmetric CDFM scaling functions have been used in the present calculations. One uses a parabolic form of the function $f_{\mathrm{RFG}, 2}^{p(n)}\left(\psi^{\prime}(R)\right)$ [Eq. (23)] at positive $\psi^{\prime}$ values, as in our previous studies on charge current neutrino scattering. The second uses an exponential form of that function at $\psi^{\prime}>0$ [Eq. (14)].

In the $\mathrm{CC}$ studies the reaction involves an incoming lepton $(v$ or $\bar{v}$ ) and the corresponding charged lepton is detected at a given angle, just as in the case of the electron scattering with incident and scattered electrons (both are $t$-channel inclusive processes). In the NC reaction, in contrast to the $\mathrm{CC}$ process, one has an incident $v$ or $\bar{v}$, but now a proton or neutron is detected at some angle, the scattered $v$ or $\bar{v}$ not being detected (this is the $u$-channel inclusive process). In this work we adopt the $u$-versus $t$-channel scaling criteria of [27] to apply the CDFM scaling functions to $u$-channel scattering at intermediate-to-high energies.

It can be seen from our results at $60^{\circ}$ that the neutrino and antineutrino cross sections are roughly in a $2: 1$ ratio. For larger scattering angle values, neutrino and antineutrino cross sections come closer, diminishing the above ratio. At forward scattering angles the $\bar{v}$ cross sections are strongly suppressed (by an order of magnitude or more). This is observed for both proton and neutron knockout. Moreover, the neutron knockout cross sections are somewhat larger than the proton knockout cross sections due to the behavior of the NC single-nucleon form factors.

It was shown that the use of asymmetric CDFM scaling function gives results which are close to those from SuSA, while the symmetric scaling function leads to a similarity with the RFG model results. The asymmetric scaling function with an exponential form [by using Eq. (14)] leads to a sharper slope of the cross sections, in comparison to that with the parabolic form [by using Eq. (23)], for the values of the kinetic energy $T_{p(n)}$ of the knocked-out nucleon smaller than those in the maximum of the cross section.

In summary, we applied the superscaling approach by means of the scaling function obtained within the CDFM (and used previously [19] for the electron and CC neutrino reactions) to the $\mathrm{NC}$ neutrino (antineutrino) scattering in the $\mathrm{QE}$ region at energy of $1 \mathrm{GeV}$ from the ${ }^{12} \mathrm{C}$ nucleus. It is pointed out that the constructed realistic CDFM scaling function is an essential ingredient in this approach for the description of the processes of lepton scattering from nuclei. Further, the CDFM model may also be useful to explore to what extent the $u$-versus $t$-channel scaling criteria, proposed in [27] on the basis of the RFG model, may be proved to hold more generally.

Another interesting future project will be to extend the scaling approach using a constructed realistic CDFM scaling function to obtain predictions for charge-changing neutrino and antineutrino scattering from nuclei in the $\Delta$-region.

\section{ACKNOWLEDGMENTS}

One of the authors (M.K.G.) is grateful for the warm hospitality given by the CSIC and for support during his stay there from the State Secretariat of Education and Universities of Spain (N/Ref. SAB2005-0012). This work was partly supported by the Bulgarian National Science Fund under Contracts No. $\Phi-1416$ and $\Phi-1501$, and by Ministerio de Educación y Ciencia (Spain) under contracts Nos. FPA200613807-C02-01, FIS2005-01105, and FIS2005-00640. 
[1] G. B. West, Phys. Rep. 18, 263 (1975).

[2] I. Sick, D. B. Day, and J. S. McCarthy, Phys. Rev. Lett. 45, 871 (1980).

[3] C. Ciofi degli Atti, E. Pace, and G. Salmè, Phys. Rev. C 36, 1208 (1987).

[4] D. B. Day, J. S. McCarthy, T. W. Donnelly, and I. Sick, Annu. Rev. Nucl. Part. Sci. 40, 357 (1990).

[5] C. Ciofi degli Atti, E. Pace, and G. Salmè, Phys. Rev. C 43, 1155 (1991).

[6] C. Ciofi degli Atti and S. Simula, Phys. Rev. C 53, 1689 (1996).

[7] C. Ciofi degli Atti and G. B. West, nucl-th/9702009.

[8] C. Ciofi degli Atti and G. B. West, Phys. Lett. B458, 447 (1999).

[9] D. Faralli, C. Ciofi degli Atti, and G. B. West, in Proceedings of 2 nd International Conference on Perspectives in Hadronic Physics, ICTP, Trieste, Italy, 1999, edited by S. Boffi, C. Ciofi degli Atti, and M. M. Giannini (World Scientific, Singapore, 2000), p. 75.

[10] W. M. Alberico, A. Molinari, T. W. Donnelly, E. L. Kronenberg, and J. W. Van Orden, Phys. Rev. C 38, 1801 (1988).

[11] M. B. Barbaro, R. Cenni, A. De Pace, T. W. Donnelly, and A. Molinari, Nucl. Phys. A643, 137 (1998).

[12] T. W. Donnelly and I. Sick, Phys. Rev. Lett. 82, 3212 (1999).

[13] T. W. Donnelly and I. Sick, Phys. Rev. C 60, 065502 (1999).

[14] C. Maieron, T. W. Donnelly, and I. Sick, Phys. Rev. C 65, 025502 (2002).

[15] M. B. Barbaro, J. A. Caballero, T. W. Donnelly, and C. Maieron, Phys. Rev. C 69, 035502 (2004).

[16] A. N. Antonov, M. K. Gaidarov, D. N. Kadrev, M. V. Ivanov, E. Moya de Guerra, and J. M. Udias, Phys. Rev. C 69, 044321 (2004).

[17] A. N. Antonov, M. K. Gaidarov, M. V. Ivanov, D. N. Kadrev, E. Moya de Guerra, P. Sarriguren, and J. M. Udias, Phys. Rev. C 71, 014317 (2005).

[18] A. N. Antonov, M. V. Ivanov, M. K. Gaidarov, E. Moya de Guerra, P. Sarriguren, and J. M. Udias, Phys. Rev. C 73, 047302 (2006).

[19] A. N. Antonov, M. V. Ivanov, M. K. Gaidarov, E. Moya de Guerra, J. A. Caballero, M. B. Barbaro, J. M. Udias, and P. Sarriguren, Phys. Rev. C 74, 054603 (2006).

[20] A. N. Antonov, M. V. Ivanov, M. K. Gaidarov, and E. Moya de Guerra, Phys. Rev. C 75, 034319 (2007).

[21] O. Benhar, D. Day, and I. Sick, nucl-ex/0603029.

[22] L. Alvarez-Ruso, M. B. Barbaro, T. W. Donnelly, and A. Molinari, Nucl. Phys. A724, 157 (2003).

[23] J. E. Amaro, M. B. Barbaro, J. A. Caballero, T. W. Donnelly, and A. Molinari, Nucl. Phys. A697, 388 (2002); A723, 181 (2003); Phys. Rep. 368, 317 (2002).

[24] A. De Pace, M. Nardi, W. M. Alberico, T. W. Donnelly, and A. Molinari, Nucl. Phys. A726, 303 (2003); A741, 249 (2004).

[25] J. E. Amaro, M. B. Barbaro, J. A. Caballero, T. W. Donnelly, and A. Molinari, Nucl. Phys. A643, 349 (1998).

[26] J. E. Amaro, M. B. Barbaro, J. A. Caballero, T. W. Donnelly, and A. Molinari, Nucl. Phys. A723, 181 (2003).
[27] J. E. Amaro, M. B. Barbaro, J. A. Caballero, and T. W. Donnelly, Phys. Rev. C 73, 035503 (2006).

[28] A. N. Antonov, V. A. Nikolaev, and I. Zh. Petkov, Bulg. J. Phys. 6, 151 (1979); Z. Phys. A 297, 257 (1980); A304, 239 (1982); Nuovo Cimento A 86, 23 (1985); A102, 1701 (1989); A. N. Antonov, D. N. Kadrev, and P. E. Hodgson, Phys. Rev. C 50, 164 (1994).

[29] A. N. Antonov, P. E. Hodgson, and I. Zh. Petkov, Nucleon Momentum and Density Distributions in Nuclei (Clarendon Press, Oxford, 1988); Nucleon Correlations in Nuclei (SpringerVerlag, Berlin-Heidelberg-New York, 1993).

[30] J. E. Amaro, M. B. Barbaro, J. A. Caballero, T. W. Donnelly, A. Molinari, and I. Sick, Phys. Rev. C 71, 015501 (2005).

[31] J. A. Caballero, J. E. Amaro, M. B. Barbaro, T. W. Donnelly, C. Maieron, and J. M. Udias, Phys. Rev. Lett. 95, 252502 (2005).

[32] J. A. Caballero, Phys. Rev. C 74, 015502 (2006).

[33] J. E. Amaro, M. B. Barbaro, J. A. Caballero, T. W. Donnelly, and J. M. Udias, Phys. Rev. C 75, 034613 (2007).

[34] Y. Fukuda et al. (The Super-Kamiokande Collaboration), Phys. Rev. Lett. 81, 1562 (1998); M. H. Ahn et al. (K2K Collaboration), ibid. 90, 041801 (2003); Q.-R. Ahmad et al. (SNO Collaboration), ibid. 87, 071301 (2001); 89, 011301 (2002); K. Eguchi et al. (KamLAND Collaboration), ibid. 90, 021802 (2003); C. Athanassopoulos et al. (LSND Collaboration), ibid. 77, 3082 (1996); 81, 1774 (1998).

[35] J. E. Amaro, M. B. Barbaro, J. A. Caballero, T. W. Donnelly, and C. Maieron, Phys. Rev. C 71, 065501 (2005).

[36] A. Meucci, C. Giusti, and F. D. Pacati, Nucl. Phys. A744, 307 (2004).

[37] M. B. Barbaro, Nucl. Phys. B, Proc. Suppl. 159, 186 (2006); nucl-th/0602011.

[38] M. C. Martinez, P. Lava, N. Jachowicz, J. Ryckebusch, J. Vantournhout, K. Udias, and J. M. Udias, Phys. Rev. C 73, 024607 (2006).

[39] J. Nieves, M. Valverde, and M. J. Vicente-Vacas, Nucl. Phys. B, Proc. Suppl. 155, 263 (2006); nucl-th/0510010.

[40] M. B. Barbaro, J. E. Amaro, J. A. Caballero, T. W. Donnelly, and A. Molinari, Nucl. Phys. B, Proc. Suppl. 155, 257 (2006); nucl-th/0509022.

[41] C. Maieron, M. C. Martinez, J. A. Caballero, and J. M. Udias, Phys. Rev. C 68, 048501 (2003).

[42] A. Meucci, C. Giusti, and F. D. Pacati, Nucl. Phys. A739, 277 (2004); A773, 250 (2006).

[43] O. Benhar, Nucl. Phys. B, Proc. Suppl. 139, 15 (2005); nuclth/0408045; O. Benhar and N. Farina, Nucl. Phys. B, Proc. Suppl. 139, 230 (2005); nucl-th/0407106; O. Benhar, N. Farina, H. Nakamura, M. Sakuda, and R. Seki, Nucl. Phys. B, Proc. Suppl. 155, 254 (2006); hep-ph/0510259; Phys. Rev. D 72, 053005 (2005)

[44] G. Co', Nucl. Phys. B, Proc. Suppl. 159, 192 (2006); nuclth/0601034; A. Botrugno and G. Co', Nucl. Phys. A761, 200 (2005); M. Martini, G. Co', M. Anguiano, and A. M. Lallena, Phys. Rev. C 75, 034604 (2007).

[45] T. Leitner, L. Alvarez-Ruso, and U. Mosel, Phys. Rev. C 73, 065502 (2006).

[46] B. Szczerbinska, T. Sato, K. Kubodera, and T.-S. H. Lee, Phys. Lett. B649, 132 (2007).

[47] A. N. Antonov, M. K. Gaidarov, M. V. Ivanov, D. N. Kadrev, G. Z. Krumova, P. E. Hodgson, and H. V. von Geramb, Phys. Rev. C 65, 024306 (2002). 
[48] J. Carbonell and V. A. Karmanov, Nucl. Phys. A581, 625 (1995).

[49] J. Carbonell, B. Desplanques, V. A. Karmanov, and J.-F. Mathiot, Phys. Rep. 300, 215 (1998).

[50] M. B. Barbaro, A. De Pace, T. W. Donnelly, A. Molinari, and M. J. Musolf, Phys. Rev. C 54, 1954 (1996).
[51] G. Höhler, E. Pietarinen, I. Sabba-Stefanescu, F. Borkowski, G. G. Simon, V. H. Walther, and R. D. Wendling, Nucl. Phys. B114, 505 (1976).

[52] V. V. Burov, D. N. Kadrev, V. K. Lukyanov, and Yu. S. Pol', Phys. At. Nucl. 61, 525 (1998).

[53] H. de Vries, C. W. de Jager, and C. de Vries, At. Data Nucl. Data Tables 36, 495 (1987). 